\title{
CARACTERÍSTICAS DE PACIENTES CON PARÁLISIS CEREBRAL ATENDIDOS EN CONSULTA EXTERNA DE NEUROPEDIATRÍA EN UN HOSPITAL PERUANO
}

\author{
Judith R. Vila1,a,b, Ivan O. Espinoza ${ }^{1,3, a, b, d}$, Daniel Guillén ${ }^{1,3, a, b, e}$, Frine Samalvides ${ }^{2,3, a, c, d}$
}

\begin{abstract}
RESUMEN
El objetivo del estudio fue describir las características de consulta neurológica y referencia de los niños con parálisis cerebral (PC). Estudio descriptivo retrospectivo. Se evaluaron las historias clínicas de los niños con PC asistentes a consulta externa de la unidad de Neuropediatría del Hospital Nacional Cayetano Heredia entre los años 2011 y 2012 . Se incluyeron 81 niños, el $53,1 \%$ fueron varones; la PC espástica fue la más frecuente $(72,8 \%)$, la encefalopatía hipóxico isquémica $(28,1 \%)$ y las malformaciones cerebrales $(28,1 \%)$ fueron las principales causas en neonatos a término. La edad media al momento del diagnóstico fue a los 4,1 $\pm 3,2$ años, la epilepsia fue el motivo más frecuente de consulta neuropediátrica. El 58\% fue hospitalizado al menos una vez. El tiempo de espera para ser atendido por terapia física tuvo una mediana de 2 meses (rango intercuartílico = 0,8 - 9). Concluimos que el diagnóstico de PC fue tardío, el tiempo de espera para acceder a la atención especializada fue prolongado. Se requieren protocolos y mayor eficiencia para atender en forma adecuada a los niños con PC.
\end{abstract}

Palabras clave: Parálisis cerebral; Atención médica; Niño; Epilepsia; Discapacidad intelectual (fuente: DeCS BIREME)

\section{CHARACTERISTICS OF PATIENTS WITH CEREBRAL PALSY SEEN IN NEU- ROPEDIATRIC EXTERNAL CONSULTATION IN A PERUVIAN HOSPITAL}

\begin{abstract}
The aim of this study was to describe the neurological consultation and baseline characteristics of children with cerebral palsy (CP). The clinical records of children with $\mathrm{CP}$ attending an external consultation of the Neuropediatric department of Hospital Nacional Cayetano Heredia between 2011 and 2012 were assessed in this retrospective descriptive study. A total of 81 children were included: $53.1 \%$ were boys. Spastic CP was the most frequent $(72.8 \%)$, hypoxic-isquemic encephalopathy $(28.1 \%)$ and cerebral malformations $(28.1 \%)$ were the main causes in term newborn. The average age at the time of diagnosis was $4.1 \pm 3.2$ years; epilepsy was the most frequent reason for neuropediatric consultation, and $58 \%$ were admitted to the hospital at least once. The waiting period to be seen by a physical therapist was around 2 months (interquartile range $=0.8-9$ ). We reached the conclusion that CP was delayed, and the waiting period to access specialized care was prolonged. Protocols and increased efficiency are required to provide adequate medical care to children with $\mathrm{CP}$.
\end{abstract}

Key words: Cerebral palsy, medical care, child, epilepsy, intellectual disability (source: MeSH NLM)

\section{INTRODUCCIÓN}

La parálisis cerebral (PC) se define como el conjunto de desórdenes del movimiento y la postura que limitan la actividad del niño y son producidos por lesiones no progresivas que ocurrieron durante el desarrollo del sistema nervioso central (SNC). Además, pueden presentar alteraciones de la sensibilidad, percepción, cognición, comunicación, conducta, y problemas psicológicos ${ }^{(1)}$.
La incidencia de PC en países europeos varía entre 2,0 a 2,5 por 1000 nacidos vivos, en Estados Unidos es 3,6 por 1000 nacidos vivos y en países en desarrollo alcanza 7,0 por 1000 nacidos vivos ${ }^{(2)}$. En el Perú, un estudio realizado en el Hospital Nacional Cayetano Heredia $(\mathrm{HNCH})$ en el año 1993, reportó una prevalencia de 5,2 por 1000 nacidos vivos ${ }^{(3)}$.

La frecuencia de niños con PC ha aumentado debido al incremento en la supervivencia de los niños prematuros

\footnotetext{
Departamento de Pediatría, Hospital Nacional Cayetano Heredia. Lima, Perú.

Departamento de Medicina, Hospital Nacional Cayetano Heredia. Lima, Perú.

Universidad Peruana Cayetano Heredia. Lima, Perú.

a Médico cirujano; ${ }^{b}$ neurólogo pediatra; ${ }^{\mathrm{c}}$ especialista en enfermedades infecciosas y tropicales; ${ }^{\mathrm{d}}$ maestría en Epidemiología Clínica; ${ }^{\mathrm{e}}$ doctor en medicina Recibido: 11/02/2016 Aprobado: 24/08/2016
} 
extremos y al descenso de la mortalidad infantil (4) y se espera que haya mayor demanda de servicios especializados en su atención.

La Academia Americana de Neurología recomienda que en la evaluación inicial de los niños con PC se confirme la naturaleza no progresiva del desorden motor y se formule el plan de trabajo para establecer la etiología, clasificar la forma clínica y determinar la presencia de disturbios asociados como retardo mental (RM), alteraciones oftalmológicas, auditivas, desórdenes del habla, nutricionales y de la función oro-motora ${ }^{(5)}$.

Las Academias Americanas de Pediatría y Parálisis Cerebral, y el Estudio de Vigilancia de Parálisis Cerebral en Europa recomiendan un manejo interdisciplinario de las condiciones motoras derivadas del trastorno postural, limitación del movimiento, escoliosis, inestabilidad articular, disfunción vesical e intestinal, disartria, disfagia, trastornos de la deglución y salud oral, así como problemas nutricionales y del crecimiento; por lo que estos pacientes deben ser referidos oportunamente para su evaluación por un equipo de profesionales que incluya al pediatra, al neurólogo pediatra, al cirujano ortopedista, el odontólogo, el nutricionista, el terapista físico, el terapista de lenguaje, los trabajadores sociales y el psicólogo ${ }^{(5-7)}$. Adicionalmente, la demanda en salud de los niños con PC se multiplica ${ }^{(8)}$ cuando se agrega a su condición una o más comorbilidades.

No existen acuerdos internacionales que indiquen de forma específica el mínimo de atenciones especializadas requeridas, y hay pocos estudios que evalúen la implementación de aquellas recomendaciones en el cuidado de la salud para los pacientes con PC y el acceso a los servicios que proveen tales atenciones ${ }^{(5,9)}$.

En el Perú no existen protocolos de atención de los niños con PC. Se requiere evidencias respecto a la magnitud de las brechas en las necesidades de salud para poder diseñar e implementar de forma óptima la atención de salud para pacientes con PC ${ }^{(10)}$. El propósito de este estudio es describir las características de consulta neurológica y referencia a las especialidades correspondientes de los niños con PC.

\section{EL ESTUDIO}

Estudio observacional, descriptivo, de corte retrospectivo. Se incluyeron a todos los pacientes menores de catorce años con diagnóstico de PC tratados en la Unidad Neuropediátrica del $\mathrm{HNCH}$ durante los años 2011-
2012. Fueron excluidos los pacientes con regresión del desarrollo o historias clínicas incompletas.

Las variables consideradas fueron: forma clínica de PC, causa atribuible de PC, edad de ocurrencia de lesión al SNC, comorbilidades relacionadas, motivo de consulta en la primera atención neuropediátrica, edad de diagnóstico de PC, atención de salud solicitada, atención de salud realizada, y tiempo de espera de atención de salud especializada, definida como el tiempo transcurrido entre la fecha registrada en la hoja de interconsulta de la atención requerida y la fecha registrada en la primera atención del servicio al que fue derivado.

Para la identificación de los pacientes, se revisaron los registros de atención diaria de consulta externa de la Unidad Neuropediátrica (hoja HIS) de los años 20112012 y se revisó, posteriormente, las historias clínicas de los pacientes seleccionados. Se revisó la información clínica de la consulta neuropediátrica en la cual se consignó por primera vez el diagnóstico definitivo de PC, y las consultas realizadas en otros servicios y especialidades a los cuales los pacientes con PC tuvieron acceso (medicina física, pediatría, odontología, neuropsicología, gastroenterología, neumología, oftalmología, y otros).

El análisis de datos fue realizado utilizando en paquete estadístico STATA (versión 11.0). Para la presentación de variables categóricas se utilizaron frecuencias absolutas y relativas, y para las variables continuas medidas de tendencia central.

El proyecto fue aprobado por los Comités de Ética en Investigación de la Universidad Peruana Cayetano Heredia y del $\mathrm{HNCH}$.

\section{HALLAZGOS}

Se identificaron 114 pacientes con diagnóstico inicial de PC, se excluyeron 28 por historias clínicas incompletas y 5 por enfermedad progresiva; finalmente, fueron seleccionados 81 casos. El 53,1\% correspondió al sexo masculino.

La edad de ocurrencia de la lesión al SNC fue principalmente perinatal (59,3\%). Hubo 64 recién nacidos a término en quienes las causas más frecuentes fueron la encefalopatía hipóxico-isquémica $(28,1 \%)$ y las malformaciones cerebrales $(28,1 \%)$, mientras que en los prematuros, la leucomalacia periventricular $(41,2 \%)$, la hemorragia intraventricular $(29,4 \%)$ y la meningitis $(11,8 \%)$ fueron las causas predominantes (Tabla 1$)$. 
Tabla 1. Características clínicas de niños con parálisis cerebral atendidos en consulta externa de la unidad de Neuropediatría del HNCH 2011-2012. Lima, Perú

\begin{tabular}{|c|c|c|}
\hline Característica & $\mathbf{N}$ & $\%$ \\
\hline \multicolumn{3}{|l|}{ Sexo } \\
\hline Masculino & 43 & 53,1 \\
\hline Femenino & 38 & 46,9 \\
\hline \multicolumn{3}{|c|}{ Edad de ocurrencia de la lesión al sistema nervioso central } \\
\hline \multicolumn{3}{|c|}{ Prenatal } \\
\hline $\begin{array}{l}\text { Desde la concepción a }<28 \\
\text { semanas }\end{array}$ & 10 & 9,9 \\
\hline Mayor o igual a 28 semanas & 37 & 49,3 \\
\hline \multicolumn{3}{|l|}{ Posnatal } \\
\hline Neonatal & 11 & 8,6 \\
\hline 1 mes - 1 año 11 meses & 16 & 19,8 \\
\hline 2 años - 4 años & 7 & 8,6 \\
\hline \multicolumn{3}{|l|}{ Posible causa atribuible } \\
\hline \multicolumn{3}{|l|}{ Recién nacido pretérmino $(n=17)$} \\
\hline Leucomalacia periventricular & 7 & 41,2 \\
\hline Hemorragia intraventricular & 5 & 29,4 \\
\hline Meningoencefalitis & 2 & 11,8 \\
\hline No identificados & 3 & 17,6 \\
\hline \multicolumn{3}{|l|}{ Recién nacido a término $(n=64)$} \\
\hline $\begin{array}{l}\text { Encefalopatía hipóxico - isqué- } \\
\text { mica }\end{array}$ & 18 & 28,1 \\
\hline Malformaciones cerebrales & 18 & 28,1 \\
\hline Infarto cerebral & 9 & 14,0 \\
\hline Meningoencefalitis & 7 & 10,9 \\
\hline Hiperbilirrubinemia & 5 & 7,8 \\
\hline Infecciones intrauterinas & 3 & 4,7 \\
\hline Sepsis neonatal & 1 & 1,6 \\
\hline No identificados & 3 & 1,2 \\
\hline
\end{tabular}

* En base a la revisión de los antecedentes perinatales y patológicos consignados en la historia clínica del paciente. $\mathrm{N}$ : Número de pacientes con parálisis cerebral (81 pacientes). $\mathrm{HNCH}$ : Hospital Nacional Cayetano Heredia.

La forma clínica más frecuente fue la PC espástica con 59 pacientes $(72,8 \%)$ y la mixta con siete $(8,6 \%)$; además, se diagnosticó epilepsia en 63 pacientes $(77,8 \%)$, RM en $41(50,6 \%)$ y caries dental en $33(40,7 \%)$.

La edad media de diagnóstico de PC fue 4,1 \pm 3,2 años, el principal motivo de consulta referido por los padres en la primera atención neuropediátrica o consignado en la hoja de interconsulta, fue la PC. Sin embargo, 19 pacientes acudieron por otros motivos diferentes a la PC. Un total de 47 pacientes (58\%) fueron hospitalizados alguna vez, siendo los principales motivos epilepsia $(40,4 \%)$ y neumonía $(21,3 \%)$ (Tabla 2$)$.

Para el 76,5\% de los pacientes (62/81) se solicitó la atención en rehabilitación, pero solo se realizó en $69,4 \%$; el tiempo de espera para la evaluación por el especialista tuvo una
Tabla 2. Características de la primera atención médica a pacientes con parálisis cerebral en la unidad de Neuropediatría del HNCH 2011-2012. Lima, Perú

\begin{tabular}{|c|c|c|}
\hline Característica & $\mathbf{N}$ & $\%$ \\
\hline \multicolumn{3}{|l|}{ Edad al momento del diagnóstico } \\
\hline$<2$ años & 32 & 39,5 \\
\hline 2 a 5 años & 21 & 25,9 \\
\hline Mayor de 5 años & 28 & 34,6 \\
\hline $\begin{array}{l}\text { Edad (media } \pm \text { desviación } \\
\text { estándar) }\end{array}$ & \multicolumn{2}{|c|}{$4.1 \pm 3.2$} \\
\hline \multicolumn{3}{|c|}{ Motivo de consulta en la primera atención neuropediátrica } \\
\hline \multicolumn{3}{|c|}{ Parálisis cerebral como principal motivo } \\
\hline $\mathrm{PC}$ & 7 & 8,6 \\
\hline $\mathrm{PC}+\mathrm{RM}$ & 6 & 7,4 \\
\hline PC + Epilepsia & 14 & 17,3 \\
\hline PC + Epilepsia + RM & 35 & 43,2 \\
\hline \multicolumn{3}{|c|}{ Pacientes que acudieron por otros motivos } \\
\hline Epilepsia & 5 & 6,2 \\
\hline Retraso del desarrollo & 10 & 12,3 \\
\hline Otros & 4 & 4,9 \\
\hline \multicolumn{3}{|l|}{ Número de hospitalizaciones } \\
\hline 1 & 26 & 32,1 \\
\hline 2 & 13 & 16,0 \\
\hline$\geq 3$ & 8 & 9,9 \\
\hline Ninguna & 34 & 42,0 \\
\hline \multicolumn{3}{|l|}{ Motivo principal de hospitalización } \\
\hline Epilepsia & 19 & 40,4 \\
\hline Neumonía & 10 & 21,3 \\
\hline Patología gastrointestinal & 7 & 14,9 \\
\hline Patología quirúrgica & 3 & 6,4 \\
\hline Otros & 4 & 8,5 \\
\hline No especificado & 4 & 8,5 \\
\hline
\end{tabular}

$\mathrm{HNCH}$ : Hospital Nacional Cayetano Heredia. N: número de pacientes con PC (81 pacientes). PC: parálisis cerebral. RM: retardo mental.

mediana de 1,5 meses (rango intercuartílico $[R I C]=1-2,7$ ), la espera para acceder a la terapia física indicada fue de 2 meses $(\mathrm{RIC}=0,8-9)$, y para acceder a terapia por trastornos del habla fue de 2,5 meses $(\mathrm{RIC}=0,5-24)$.

Respecto a las interconsultas hacia otras especialidades, el $33,3 \%$ fue referido a oftalmología, y el 66,7\% (18/27) efectivizó la referencia. La mediana de tiempo de espera de 3 meses ( $\mathrm{RIC}=1-36)$. Entre los diagnósticos destacaron el estrabismo $(61,1 \%)$ y déficit de agudeza visual $(33,3 \%)$.

El $50,6 \%$ de los pacientes fue diagnosticado con RM. De ellos, solo el $32,1 \%$ de los pacientes con PC fue referido a Neuropsicología, de los cuales el 76,9\% (20/26) alcanzó dicha evaluación. De los 20 pacientes evaluados, el $60 \%$ fue diagnosticado con RM severo y el $40 \%$ con RM moderado. La mediana de tiempo de espera fue de 2 meses (RIC=0,8-36). 
Se solicitó la atención odontológica en $28,4 \%$ de pacientes, dicha atención se cumplió en $43,5 \%$, y la mediana de tiempo de espera fue de 20,7 meses (RIC=7,6-66).

El menor cumplimiento de atención solicitada ocurrió en nutrición $13,3 \%$ (2/15), y el tiempo de espera registrado fue de 6 meses. Ambos pacientes tenían el diagnóstico de desnutrición severa. En los demás pacientes no se logró establecer la condición nutricional por no ser una actividad rutinaria en el consultorio.

En ningún paciente se registró la solicitud de atención interdisciplinaria en todos los servicios de salud requeridos. No se logró identificar el tiempo de espera para la primera consulta neuropediátrica pues la fuente de investigación no lo registraba. Los demás resultados se muestran en las tablas 3 y 4 .

Tabla 3. Atenciones solicitadas y realizadas para la referencia de los pacientes con parálisis cerebral atendidos en la unidad de Neuropediatría del HNCN, 2011-2012. Lima, Perú

\begin{tabular}{|c|c|c|c|c|}
\hline \multirow[t]{2}{*}{ Consulta externa } & \multicolumn{2}{|c|}{$\begin{array}{l}\text { Atención soli- } \\
\text { citada }\end{array}$} & \multicolumn{2}{|c|}{$\begin{array}{c}\text { Atención reali- } \\
\text { zada }\end{array}$} \\
\hline & $\mathbf{N}$ & $\%$ & $\mathbf{N}$ & $\%$ * \\
\hline Medicina física & 62 & 76,5 & 43 & 69,4 \\
\hline $\begin{array}{l}\text { Atención por trastorno } \\
\text { del habla }\end{array}$ & 17 & 21,0 & 06 & 35,3 \\
\hline Oftalmología & 27 & 33,3 & 18 & 66,7 \\
\hline Neuropsicología & 26 & 32,1 & 20 & 76,9 \\
\hline Salud oral & 23 & 28,4 & 10 & 43,5 \\
\hline Audiología & 18 & 22,2 & 8 & 44,4 \\
\hline Pediatría & 16 & 19,8 & 3 & 18,8 \\
\hline Nutrición & 15 & 18,5 & 2 & 13,3 \\
\hline $\begin{array}{l}\text { Atención por trastorno } \\
\text { oromotor }\end{array}$ & 4 & 4,9 & 0 & 0,0 \\
\hline Neumología & 4 & 4,9 & 1 & 25,0 \\
\hline Psicología & 3 & 3,7 & 1 & 33,3 \\
\hline Nefrología & 3 & 3,7 & 2 & 66,7 \\
\hline Traumatología & 3 & 3,7 & 2 & 66,7 \\
\hline Otros † & 16 & 19,7 & 7 & 43,8 \\
\hline
\end{tabular}

* Sobre el total de atenciones solicitadas. Atención solicitada: solicitud de atención especializada realizada por neuropediatra. Atención realizada: atención efectuada en otros servicios de salud registrada en la historia clínica; † Otros: Psiquiatría $=2$, Hematología $=2$, Cardiología $=2$, Gastroenterología $=2$, Neurocirugía $=1$, Cirugía Máxilo facial $=1$, Genética $=1$, Endocrinología, Cirugía Pediátrica =1, Urología = 1, Otorrinolaringología $=1$, Servicio Social $=1$. HNCH: Hospital Nacional Cayetano Heredia.
Tabla 4. Tiempo de espera para la atención de niños con $\mathrm{PC}$ en otros servicios de salud solicitados por la unidad de Neuropediatría del $\mathrm{HNCH}, 2011-2012$. Lima, Perú

\begin{tabular}{lccc}
\hline & & \multicolumn{2}{c}{$\begin{array}{c}\text { Tiempo de espera } \\
\text { (en meses) }\end{array}$} \\
\cline { 3 - 4 } Destino de referencia & $\mathbf{N}$ & Mediana & $\begin{array}{c}\text { Rango } \\
\text { intercuartílico }\end{array}$ \\
\hline Terapia física & 43 & 2,0 & $0,8-9,0$ \\
Neuropsicología & 20 & 2,0 & $0,8-36$ \\
Oftalmología & 18 & 3,0 & $1,0-36$ \\
Salud oral & 10 & 20,7 & $7,6-66$ \\
Audiología & 08 & 6,0 & $2,0-11$ \\
Atención por trastorno & 06 & 2,5 & $0,5-24$ \\
del habla & 03 & 0,7 & $\mathrm{NA}$ \\
\hline Pediatría & 02 & 6,0 & $\mathrm{NA}$ \\
\hline Nutrición & 13 & 02 & $1,5-2.0$ \\
\hline Otros * & & & \\
\hline
\end{tabular}

* Traumatología $=2$, Hematología $=2$, Cardiología = 2, Gastroenterología $=1$, Neurocirugía $=1$, Cirugía Pediátrica $=1$, Neumología $=1$, Psicología $=1$, Nefrología $=1 . \mathrm{NA}=\mathrm{No}$ aplica. $\mathrm{HNCH}$ : Hospital Nacional Cayetano Heredia.

\section{DISCUSIÓN}

En nuestra serie, a pesar de que la presunción de causalidad de los factores estudiados es solo aproximada, encontramos diferencias en cuanto a la etiología. Mientras que Bancalari identificó a la encefalopatía hipóxico-isquémica como causa perinatal de $\mathrm{PC}$ en el $49 \%$ de los casos ${ }^{(3)}$ lo hallado en nuestro estudio es menor; este cambio se debería a mejoras en la atención perinatal en las dos últimas décadas y la necesidad de precisar la etiología del recién nacido con encefalopatía. Las formas clínicas más frecuentes, la PC espástica seguida de la PC mixta, son similares a otros estudios $^{(7,11,12)}$.

El diagnóstico clínico de PC es confiable a partir de los 18 meses ${ }^{(13)}$. En nuestro estudio solo el 39,5\% tenía menos de dos años de edad al momento del diagnóstico. Esta demora es superior a lo encontrado en Turquía para el año de $1972(77,4 \%)$ o para el año de 1994 (91,9\%) (11). La accesibilidad limitada a la atención especializada de niños con riesgo neurológico, la falta de sospecha clínica por parte de los médicos generales y otros profesionales de la salud explicarían estas diferencias ${ }^{(14)}$.

Shevell en Canadá, reportó un tiempo promedio de 15,5 meses transcurrido desde la preocupación inicial de los padres hasta la realización efectiva de la atención médica especializada; retraso que se debería a que los padres no reconocen las dificultades motoras, no comunican sus preocupaciones a los trabajadores de salud, o porque el profesional a cargo adopta una actitud expectante ${ }^{(15)}$. Si bien nuestro estudio no pudo determinar el tiempo de retraso en el diagnóstico, esta variable debería ser incluida en próximas investigaciones. 
Con respecto a las comorbilidades; la epilepsia acompaña frecuentemente a la PC entre el $28 \%$ y el $50 \%$ de los casos, y el retardo mental en el $52 \%{ }^{(5)}$. En nuestro estudio, un grupo de niños que buscaron atención por epilepsia fueron también diagnosticados de PC y/o RM y viceversa.

En nuestra serie, aproximadamente la mitad de los pacientes con $\mathrm{PC}$ accedió a terapia física, proporción que es inferior al $75,3 \%$ reportado por Tosun (11). Majnemer, en un estudio desarrollado en Canadá, reportó que el $84,6 \%$ de los pacientes con PC utilizaron, incluso, más de un servicio de rehabilitación ${ }^{(10)}$. En nuestro caso, no pudimos identificar el porcentaje de atención en otros servicios de rehabilitación y las terapias específicas que recibieron los pacientes.

A pesar de que los problemas oftalmológicos pueden estar presentes entre el $50 \%$ y el $90 \%$ de los pacientes con PC ${ }^{(16)}$, en nuestra serie encontramos que solo un tercio de los pacientes fueron referidos a oftalmología y solo $66,7 \%$ de estos cumplió con dicha evaluación. Esta falta de evaluación oftalmológica fue observada también por Ozturk en Turquía, quien reportó, en una serie de 194 niños con PC espástica y edades comprendidas entre los 6 meses y los 18 años, que el 47,9\% no tenía evaluación oftalmológica previa ${ }^{(16)}$. Tanto en el estudio de Ozturk como en el nuestro los problemas oftalmológicos más frecuentes fueron los errores de refracción y el estrabismo.

Existen estudios que demuestran que la evaluación cognitiva en niños con PC es posible y se ha identificado que la media del coeficiente intelectual de los niños con PC es más bajo en comparación con la población general ${ }^{(17)}$. En nuestro estudio, a pesar de que no todos los niños identificados con RM fue derivado a neuropsicología, el $76,9 \%$ de los niños referidos sí logró ser evaluado por esta especialidad, probablemente porque uno de los motivos principales para solicitar esta evaluación fue el déficit cognitivo y la consulta neurológica es justamente la necesidad de los padres de evaluar el estado cognitivo del paciente para definir sus opciones de escolaridad.

En la mayoría de los registros de las consultas neuropediátricas no se consignó ni el peso ni la talla de los pacientes, y no fue posible conocer la proporción de los niños con malnutrición. Dicha omisión podría deberse a la dificultad para realizar mediciones antropométricas a pacientes con PC o a la falta de instrumentos que faciliten esta actividad.

Se debe resaltar la escasa proporción de pacientes que acudieron a la consulta nutricional (2/15), a pesar de que la prevalencia de desnutrición en niños con $\mathrm{PC}$ es muy alta, tal como se indica en un estudio desarrollado en el 2006 en Lima - Perú que reportaba que el 81,1\% de pacientes con PC estaba desnutrido ${ }^{(18)}$.

La prevalencia de problemas al alimentarse se correlaciona directamente con la severidad de compromiso motor, y el compromiso de las habilidades para la alimentación son uno de los factores pronósticos de supervivencia ${ }^{(19)}$. No tenemos datos acerca del acceso a terapias específicas para mejorar la masticación y/o la deglución, pero consideramos que deben formar parte de los protocolos de atención hospitalaria.

En nuestra serie encontramos que la frecuencia de caries fue del $40,7 \%$, valor inferior a lo reportado por Soto, quien halló una frecuencia de $92,4 \%$ caries en una muestra de 170 niños con PC ${ }^{(20)}$. Creemos que nuestro porcentaje corresponde a un subregistro. Solo se solicitó atención odontológica al $28,4 \%$ de los pacientes y una proporción menor alcanzó tal atención. Tal información es similar a lo reportado por Oliveira, quién observó que solo el $12,6 \%$ de niños con PC habían sido evaluados por odontología a los 3 años de edad, y que la principal causa referida por los cuidadores era la falta de profesionales capacitados en el manejo de estos pacientes; aunque también podría deberse al desconocimiento de los cuidadores acerca de la importancia del diagnóstico temprano ${ }^{(21)}$.

Llama la atención el bajo porcentaje de pacientes con PC que realizan por lo menos una consulta de pediatría lo cual contribuye a la inadecuada identificación de los problemas nutricionales, odontológicos, inmunizaciones entre otros.

El intervalo de espera para el acceso a la atención interdisciplinaria iba de 1 día hasta 84 meses. Tal disparidad demostraría que intervienen factores externos al sistema de salud, además de dificultades al acceso a una atención de salud especializada. Entre tales factores la educación de los padres y la condición socioeconómica cumplen un rol importante. El retraso en las evaluaciones especializadas es crítico, pues dificultan la detección temprana y el manejo oportuno de comorbilidades que afectarían al desempeño motor y cognitivo del niño, así como su bienestar ${ }^{(14,22)}$.

Las limitaciones del estudio se derivan del uso de fuentes secundarias: una cantidad considerable de historias clínicas fueron excluidas por tener información incompleta. Además, algunas variables, como la evaluación nutricional y odontológica, estaban consignadas en pocas atenciones, por lo que los porcentajes podrían no reflejar completamente la realidad. Esto dificulta la valoración del proceso de atención integral del paciente así como la verdadera satisfacción de sus necesidades. 
A pesar de las limitaciones descritas, creemos que este trabajo ilustra las dificultades en nuestro sistema de salud y da una visión real de la problemática actual en la atención de los niños con PC. Es necesario desarrollar otros estudios que permitan conocer la prevalencia actual de la PC, mejorar la calidad de atención y formular directrices nacionales que orienten la atención de salud de niños con PC.

Concluimos que el diagnóstico de PC fue tardío, la evaluación de los pacientes fue incompleta y no coordinada, y los tiempos de espera para acceder a la atención especializada fueron prolongados.

Fuentes de financiamiento: autofinanciado.

Conflictos de interés: los autores declaran no tener conflictos de interés.

Contribución de autoría: JRV, IOE, FS participaron en el diseño de estudio; JRV, IOE en la adquisición de datos; JRV IOE, DG en el análisis e interpretación de datos; JRV, IOE, FS, DG redactaron del manuscrito; IOE, FS, DG revisaron críticamente el manuscrito; JRV realizó el análisis estadístico, IOE dio el apoyo administrativo, logístico y técnico.

\section{REFERENCIAS BIBLIOGRÁFICAS}

1. The definition and classification of cerebral palsy. Dev Med Child Neurol. 2007;49(s109):1-44. doi: 10.1111/j.1469-8749.2007.00001.x

2. Hurley DS, Sukal-Moulton T, Msall ME, Gaebler-Spira D, Krosschell KJ. The cerebral palsy research registry: development and progress toward national collaboration in the United States.JChild Neurol.2011;26(12):153441. doi: 10.1177/0883073811408903

3. Bancalari EM. Parálisis cerebral: correlato clínico-etiológico. [tesis para obtener el grado de especialista]. Lima: Universidad Peruana Cayetano Heredia, Facultad de Medicina. 1993.

4. Larroque B, Ancel P, Marret S, Marchand L, André M, Arnaud C and Kaminski M. Neurodevelopmental disabilities and special care of 5-year-old children born before 33 weeks of gestation (the EPIPAGE study): a longitudinal cohort study. Lancet. 2008;371(9615):813-20. doi: 10.1016/S0140-6736(08)60380-3

5. Ashwal S, Russman BS, Blasco PA, Miller G, Sandler A, Shevell M, et al. Practice parameter: diagnostic assessment of the child with cerebral palsy. Report of the Quality Standards Subcommittee of the American Academy of Neurology and the Practice Committee of the Child Neurology Society. Neurology. 2004;62(6):851-63. doi: 10.1212/01. WNL.0000117981.35364.1B

6. Novak I, Mcintyre S, Morgan C, Campbell L, Dark L, Morton N and Goldsmith, S. A systematic review of interventions for children with cerebral palsy: state of the evidence. Dev Med Child Neurol. 2013;55(10):885-910. doi: $10.1111 / \mathrm{dmcn} .12246$

7. Fairhurst, C. Cerebral palsy: the whys and hows. Arch Dis Child Educ Pract Ed. 2012;97(4):122-31. doi: 10.1136/ edpract-2011-300593

8. Jackson KE, Krishnaswami S, McPheeters M. Unmet health care needs in children with cerebral palsy: a cross-sectional study. Res Dev Disabil. 2011;32(6):2714 23. doi: 10.1016/j.ridd2011.05.040

9. National Institute for Health and Care Excellence (NICE). Spasticity in under 19s: management. Clinical guideline. [internet]. Manchester: NICE; 2012 [citado el 23 de diciembre de 2015]. Disponible en: https:// www.nice.org.uk/guidance/cg145/ resources/spasticity-in-under-19smanagement-35109572514757.

10. Majnemer A, Shikako-Thomas K, Lach L, Shevell M, Law M, Schmitz N et al. Rehabilitation service utilization in children and youth with cerebral palsy. Child Care Health Dev. 2014;40(2):275 8g2. doi: 10.1111/cch.12026.

11. Tosun A, Gökben S, Serdaroğlu G, Polat $\mathrm{M}$, Tekgül H. Changing views of cerebral palsy over 35 years: the experience of a center. Turk J Pediatr. 2013;5(1) :8-15.

12. McIntyre S, Taitz D, Keogh J, Goldsmith $S$, Badawi N, Blair E. A systematic review of risk factors for cerebral palsy in children born at term in developed countries. Dev Med Child Neurol. 2013;55(6):499508. doi: $10.1111 / \mathrm{dmcn} .120117$

13. Bosanquet M, Copeland L, Ware R, Boyd R. A systematic review of tests to predict cerebral palsy in young children. Dev Med Child Neurol. 2013;55(5):418-26. doi: $10.1111 / \mathrm{dmcn} .12140$

14. Shevell M, Ashwal S, Donley D, Flint J, Gingold M, Hirtz D, et al. Practice parameter: evaluation of the child with global developmental delay. Report of the Quality Standards Subcommittee of the American Academy of Neurology and The Practice Committee of the Child Neurology Society. Neurology. 2003;60(3):367-80. doi: 10.1212/01. WNL.0000031431.81555.16

15. Shevell MI, Majnemer A, Rosembaum P, Abrahamowicz M. Profile of referrals for early childhood developmental delay to ambulatory subspecialty clinics. J Child Neurol. 2001;16(9):645-50. $10.1177 / 088307380101600904$
16. Ozturk AT, Berk AT, Yaman A. Ocular disorders in children with spastic subtype of cerebral palsy. Int J Ophthalmol. 2013;6(2):204-210. doi: 10.3980/j. issn.2222-3959.2013.02.19

17. Sigurdardottir S, Eiriksdottir A, Gunnarsdottir E, Meintema M, Arnadottir U, Vik T. Cognitive profile in young Icelandic children with cerebral palsy. Dev Med Child Neurol. 2008;50(5):357-62. doi: 10.1111/j.1469-8749.2008.02046.x.

18. Del Águila A, Áibar P. Características nutricionales de niños con parálisis cerebral. ARIE-Villa El Salvador. An Fac Med. 2006;67(2):108-19. doi: 10.15381/ anales.v67i2.1248

19. Dahlseng $M O$, Finbråten $A K$, Júlíusson PB, Skranes J, Andersen G, Vik T. Feeding problems, growth and nutritional status in children with cerebral palsy. Acta Paediatr. 2012;101(1):92-8. doi:10.1111/j.16512227.2011.02412.x

20. Soto R, Vallejos R, Falconí E, Monzón F. Patologías bucales en niños con encefalopatía Infantil en el Perú. Rev Estomatol Herediana. 2006;16(2):115-9. doi: 10.20453/reh.v16i2.1915

21. Oliveira AC, Tornisiello CR. Condições de saúde e acesso ao tratamento odontológico de pacientes com paralisia cerebral atendidos em um centro de referência do Nordeste - Brasil. Rev CEFAC. 2012;14(5):861-71. doi: 10.1590/S1516-18462012005000045

22. Scherzer AL, Chhagan M, Kauchali $S$, Susser E. Global perspective on early diagnosis and intervention for children with developmental delays and disabilities. Dev Med Child Neurol. 2012;54(12):1079-84. doi: 10.1111/j.1469-8749.2012.04348.x.

Correspondencia: Judith Raquel Vila Paucarcaja

Dirección: Av. Honorio Delgado 262. Lima, Perú Teléfono: (0051) 970067325

Correo electrónico: judith.vipa@gmail.com 